\title{
SINERGIA
}

REVISTA DO INSTITUTO DE CIÊNCIAS ECONÔMICAS, ADMINISTRATIVAS E CONTÁBEIS (ICEAC)

\section{A DEMANDA POR ENERGIA ELÉTRICA RESIDENCIAL PARA O RIO GRANDE DO SUL: UMA ABORDAGEM DE DADOS EM PAINEL}

NATHIÉLE HELLWIG LIERMANN DANIEL DE ABREU PEREIRA UHR* JULIA GALLEGO ZIERO UHR***

\section{RESUMO}

O objetivo desta pesquisa é identificar a elasticidade do preço da demanda por energia elétrica do setor residencial para o Rio Grande do Sul. Para a identificação da elasticidade, utilizamos diferentes estratégias, considerando os dados na estrutura de painel: dados empilhados, efeitos aleatórios, efeitos fixos, efeitos fixos e variáveis instrumentais internas, e, por fim, efeitos fixos com variável instrumental externa. Os dados compreendem informações municipais entre os anos de 2007 a 2015. Os resultados mostram que o aumento do preço da energia elétrica residencial em $1 \%$ afeta, negativamente. a demanda em $0,46 \%$, ou seja, pode-se afirmar que a demanda por energia elétrica residencial é inelástica ao preço.

Palavras-Chaves: Eletricidade, Elasticidade-preço, Consumo de Energia.

\section{ABSTRACT}

The objective of this research is to identify the price elasticity of demand for residential sector in Rio Grande do Sul. We use different econometric strategies to identify the elasticity considering the panel data structure: pooled OLS data, random effects, fixed effects, effects fixed and internal instrumental variables, and, finally, fixed effects with external instrumental variable. The data comprise municipal information between the years 2007 to 2015 . The results show that the $1 \%$ increase in the price of residential electricity negatively affects the demand by $0.46 \%$, that is, it can be said that the demand for residential electricity is inelastic at price.

Keywords: Electricity, Price elasticity, Energy Consumption.

Recebido em: 27-05-2020 Aceito em: 16-10-2020

\section{INTRODUÇÃO}

A energia elétrica é um fator importante para o crescimento econômico e para o bem-estar individual. Uma estimativa consistente dos parâmetros da demanda de energia elétrica residencial proporciona informações relevantes para a identificação de demandas futuras. Além de ser um bom instrumento para o planejamento de políticas públicas de preços, é, também, importante, nas políticas empresariais, para a previsão de custos, já que eles identificam as reações das unidades consumidoras às variações nas tarifas.

O objetivo desse estudo é identificar a elasticidade do preço da demanda de energia residencial para o Rio Grande do Sul. Para tal, utilizamos dados municipais contemplando 497 municípios, entre os anos de 2007 a 2015. Para a identificação da elasticidade da demanda por energia elétrica residencial, utilizamos diferentes estratégias baseadas na estrutura de dados em painel: (i) dados empilhados, (ii) efeitos aleatórios, (iii) efeitos fixos, (iv) efeitos fixos e variáveis instrumentais internas, e, por fim, (v) efeitos fixos com variável instrumental externa.

Embora haja muitas publicações sobre a elasticidade preço da demanda para o Brasil (VILLAREAL; MOREIRA, 2016; ANDRADE; LOBÃO, 1997; VIANA; SILVA, 2014; MODIANO, 1984). Poucos estudos analisam a elasticidade preço da demanda para estados ou para regiões brasileiras (UHR; UHR; CHAGAS, 2017b; IRFFI et al., 2009; SIQUEIRA et al., 2006; MATTOS; LIMA, 2005). Portanto, este trabalho inova a

\footnotetext{
"Discente do Curso de Graduação em Ciências Econômicas da Universidade Federal de Pelotas.

"Pós-Doutorado em Economia pela Universidade de São Paulo (FEA/USP). Doutor em Economia pela Universidade de Brasília (UnB). Mestre em Economia pela Universidade de Brasília (UnB). Professor Associado do Departamento de Economia da Universidade Federal de Pelotas (Decon / UFPel). E-mail: daniel.uhr@gmail.com.

${ }^{* * *}$ Pós-doutora em Economia pela Universidade de São Paulo (USP). Doutora e Mestra em Economia pela Universidade de Brasília (UnB). Professora adjunta do departamento de Economia da Universidade Federal de Pelotas (UFPel).
} 
literatura porque é o primeiro trabalho que estima a elasticidade preço da demanda por energia elétrica para o Rio Grande do Sul, considerando as técnicas aplicadas e os dados utilizados. Os resultados são muito importantes para diversos setores da sociedade. Como, por exemplo, os ofertantes de bens e serviços residenciais, os quais têm a energia elétrica, podem gerir melhor sua estratégia de ação enquanto as empresas distribuidoras e geradoras (tanto públicas quanto privadas) de energia podem utilizar os resultados encontrados como um previsor da resposta demanda por energia elétrica residencial quando ocorrer alteração dos preços da energia no Rio Grande do Sul, e, assim, ajustar a sua estrutura operacional de maneira mais precisa.

De forma geral, os resultados preliminares com as técnicas tradicionais de dados em painel apresentam elasticidades negativas e estatisticamente significativas para todos os modelos, variando entre $-0,105$ e $-3,4$. O segundo conjunto de estratégias manteve o uso dos efeitos fixos e adicionou os instrumentos internos de diversas formas. Os resultados permaneceram negativos e estatisticamente significativos, com magnitudes entre $-0,145$ e 0,9 . Por fim, a estratégia que considera os efeitos fixos e utiliza como instrumento externo a variação exógina sobre diferentes regiões nos preços mostra que a elasticidade preço da demanda por energia elétrica residencial no rio Grande do sul é de $-0,46$.

Este trabalho está estruturado em seis seções, iniciando por essa introdução. A seção subsequente faz-se a revisão de literatura, seguido pela apresentação dos dados e do método de identificação. Na quinta seção, apresentam-se os resultados obtidos, e por fim, as considerações finais.

\section{REGULAMENTAÇÃO DA ENERGIA ELÉTRICA E REVISÃO DA LITERATURA}

\subsection{Divisão Regional da Geração e Distribuição de Energia}

Em 1ํ de fevereiro de 1943, foi instituída a CEEE (Companhia Estadual de Energia Elétrica), chamada na época Comissão Estadual de Energia Elétrica (uma das primeiras geradoras e distribuidoras de energia do estado do Rio Grande do Sul). Em 1952, foi considerada autarquia para atividades que compreendem a geração e a transmissão de energia elétrical. Em 1963, passou-se a chamar Companhia Estatual de Energia Elétrica, sendo responsável pela geração e pela distribuição de energia elétrica em $89 \%$ do território gaúcho (CEEE, 2015). No ano de 1997, mediante uma Assembleia Geral Extraordinária, foi autorizada a criação de três companhias de distribuição, a Companhia de Geração Térmica de Energia Elétrica, Companhia CentroOeste de Distribuição de Energia Elétrica e a Companhia Norte-Nordeste de Distribuição de Energia Elétrica. No mesmo ano, foi realizado um leilão dessas companhias.

A Companhia Centro-Oeste foi vendida à AES Guaíba, a qual, posteriormente, foi denominada AES Sul Distribuidora Gaúcha de Energia S/A (AES Sul). A Companhia Norte-Nordeste foi vendida pelo consórcio formado pela VBC (Votorantim, Bradesco e Camargo Correa), a Previ (fundo de pensão dos funcionários do Banco do Brasil) e Community Energy Alternatives, a qual passou a ser chamada Rio Grande Energia S/A (RGE). Já em 1998, a Companhia de Geração Térmica de Energia Elétrica passou a pertencer ao governo federal em troca de pagamentos de dívidas. Por fim, a CEEE assumiu o controle das atividades de geração e distribuição de energia elétrica no Sul e no Sudeste do estado. Ela passou a ser responsável pela distribuição da energia elétrica em 72 municípios (ANEEL, 2008b), que abrange a região metropolitana, incluindo a grande capital, a região do litoral, da Serra Gaúcha e a região sul, cuja área abrange $73.627 \mathrm{~km}^{2}$, representando cerca de $34 \%$ do consumo do Estado (CEEE, 2015).

A AES Sul Distribuidora Gaúcha de Energia (AES Sul) foi fundada no dia 28 de junho de 1998; era, inicialmente, chamada de Concessionária Centro-Oeste de Distribuição de Energia Elétrica, que fazia parte da Companhia Estadual de Energia Elétrica (CEEE), posteriormente, em outubro de 1997, ela foi comprada pelo grupo privado AES Corporation, por intermédio de sua subsidiária a AES Guaíba, tornando-se uma concessionária de direito privado e capital aberto, controlada pelo grupo norte americano AES (AES Sul, 2006; AES Sul, 2016; ANEEL, 2008a).

A AES Sul era responsável por atender 118 municípios gaúchos, cujo área corresponde a $99.512 \mathrm{~km}^{2}$ (ANEEL, 2008a). Ela atendia municípios da fronteira norte, da fronteira sul, da região central, do vale e da região metropolitana, ou seja, fornecia energia elétrica entre a região metropolitana de Porto Alegre até municípios do extremo oeste, como Uruguaiana e Santana do Livramento (AES Sul, 2016). A AES Sul fornecia energia para cerca de 1,3 milhão de unidades consumidoras, o que representava cerca de $30,4 \%$ da energia consumida do estado do Rio Grande do Sul (AES Brasil, 2015). O volume de vendas da AES Sul era de 9 mil GWh e possuía cerca de 3,02 bilhões em receita líquida no ano de 2015".

Em 2016, a AES Sul foi vendida para o grupo CPFL Energia, o mesmo grupo responsável pela distribuidora RGE, e passou a ser chamada de RGE Sul. O valor final da transação foi de 2,8 bilhões de reais, no qual se inclui o valor das dívidas da concessionária".

A Rio Grande Energia (RGE) resulta da venda da Companhia Centro-Oeste, que, inicialmente, pertencia à CEEE. Adquirida em outubro de 1997, passou a ser chamada de RGE no mesmo ano. Esta ficou 
responsável pelo fornecimento de energia elétrica em 254 municípios do Rio Grande do Sul, a qual corresponde a uma área de concessão equivalente a $90,1 \mathrm{mil} \mathrm{km}^{2}$, o que corresponde a $51 \%$ do território gaúcho (ANEEL, 2008c).

A RGE faz parte, desde 2006, do grupo CPFL Energia, o qual é o maior grupo privado de geração de energia elétrica do Brasil, atendendo estados como Rio Grande do Sul, São Paulo, Minas Gerais e Paraná, representando cerca de 7,5 milhões de clientes em todo o Brasill".

Após a CPFL Energia incorporar a AES Sul, passou a atender cerca de 9,1 milhões de clientes, uma área correspondente a 304 mil km² II. Passou, assim, a atender 381 municípios gaúchos, totalizando 2,86 milhões de clientes, que correspondem a cerca de $65 \%$ do total do estado, ou seja, $189 \mathrm{mil} \mathrm{km}^{2}$ de extensãolII.

Figura 1 - Mapa de Distribuição de Energia Elétrica

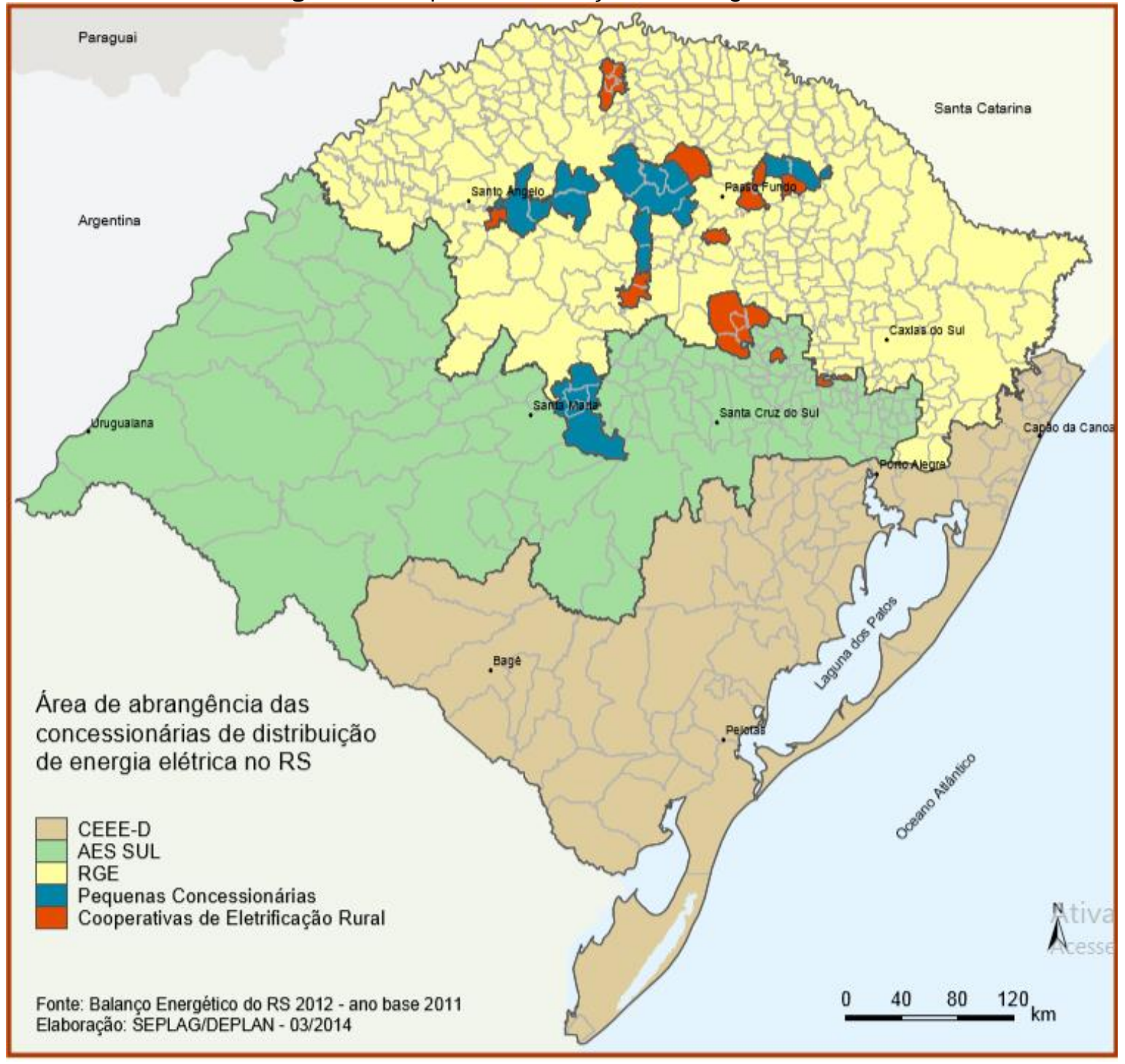

\subsection{A Definição dos Preços de Energia Elétrica no Rio Grande do Sul}

As tarifas aplicáveis na prestação de serviços, entre a AES Sul e a RGE, são muito semelhantes. O contrato de concessão № 12/97 assinado entre a União e a AES Sul e o contrato de concessão № 13/97, assinado entre a União e a RGE, ocorreram no mesmo ano, em 1997. Ambos utilizam a mesma fórmula e o mesmo padrão para a realização do cálculo. Segundo o contrato de concessão, a concessionária ficaria responsável por ajustar as tarifas anualmente, seguindo a seguinte regra: para o reajuste tarifário, a receita da concessionária seria dividida em duas parcelas que correspondem à Parcela $A$ e à Parcela B (MME, 1997a; MME, 1997b).

A Parcela A são os custos de cota da RGR - Reserva Global de Reversão-, cotas da CCC - Conta de Consumo de Combustíveis -, encargos da compensação financeira pela utilização de recursos hídricos e os decorrentes da compra de energia elétrica para revenda (MME, 1997a; MME, 1997b). E a Parcela B é o 
restante da receita após a dedução da Parcela A e do ICMS (MME, 1997a; MME, 1997b).

$$
I R T=\frac{V P A 1+V P B O(I V I \pm X)}{R A O}
$$

VPA1 é o valor da Parcela A vigente na data do reajuste e a energia comprada em detrimento ao Mercado de Referência (mercado de energia garantida nos doze meses precedentes ao reajuste). RA0 é a receita anual, não incluindo o ICMS, vigente na "data de referência anterior" e o "mercado de referência"; IVI é o número índice obtido pela divisão dos índices do IGPM da Fundação Getúlio Vargas do mês anterior ao reajuste; X é um número índice definido pelo poder concedente, e VPBO é o valor da Parcela B, levando em conta as obrigações vigentes na "data de referência" anterior e o "mercado de referência", que pode ser calculado da seguinte forma (MME, 1997a; MME, 1997b):

$$
V P B O=R A O-V P A O
$$

Sendo que VPA0 é o valor da Parcela A, considerando-se as obrigações vigentes na "data de referência anterior" e o "mercado de referência" (MME, 1997a; MME, 1997b).

$\mathrm{Na}$ AES Sul, em 2005, foi realizada a primeira alteração contratual na fórmula tarifária. Neste aditivo, passou-se a incluir componentes financeiros na Parcela $A$, como o repasse da Conta de Variação de Itens ${ }^{\mathrm{V}}$ (ANEEL, 2005a). O mesmo ocorreu para a RGE no mesmo ano (ANEEL, 2005b).

Em 2010, a AES Sul realizou outra alteração no ajuste tarifário. O objetivo deste aditivo foi neutralizar os encargos sobre a Parcela $A$, evitando que variações de mercado possam gerar receitas indevidas $\vee$ (ANEEL, 2010a), ocorrendo, simultaneamente, com a CEEE e com a RGE (ANEEL, 2010b; ANEEL, 2010c).

Já a CEEE possui um método um pouco diferenciado para cálculo do reajuste das tarifas de energia elétrica. Ele é definido pela seguinte fórmula (MME, 2000):

$$
F R=\frac{V P A_{1}+\left(V P B_{0} . I P\right)}{R A}
$$

FR é o fator de reajuste dos preços. VPA 1 é o valor dos tributos relativo ao período de referência vigente na data de referência anterior. RA é o somatório dos faturamentos de energia e de demanda em relação ao período de referência, calculados na data de referência anterior, excluindo o ICMS (MME, 2000). E IP é o mesmo índice IVI utilizado pela AES Sul e RGE. Já o $\mathrm{VPB}_{0}$ pode ser calculado pela seguinte equação (MME,2000):

$$
V P B_{0}=R A-V P A_{0}
$$

$\mathrm{VPA}_{0}$ é o valor dos tributos relativos ao período de referência vigente na data de referência em processamento (MME, 2000). Em 2005, a CEEE fez sua primeira alteração na elaboração da tarifa. Ela passou a cobrar as tarifas homologadas pelas ANEEL, a qual passa a ter a mesma fórmula que a AES Sul e a RGE em 2005 (ANEEL, 2005c).

A incidência das alíquotas de impostos sobre preço da energia elétrica é elaborada de acordo com o valor da tarifa de energia elétrica publicada pela Agência Nacional de Energia Elétrica (ANEEL) e as alíquotas de impostos. As três distribuidoras analisadas seguem o mesmo modelo. Inicialmente, deve-se aplicar a seguinte fórmula (ANEEL 2008a; ANEEL, 2008b; ANEEL, 2008c):

$$
\text { Valor a ser cobrado }=\frac{\text { Valor da tarifa publicada pela ANEEL }}{1-(\text { IIS }+ \text { COFINS }+ \text { ICMS })}
$$

O valor da tarifa publicada pela ANNEL consiste no valor do kWh estabelecido pela ANEEL referente ao período em questão. Para cálculo do PIS, é realizada uma média, bem como uma média do COFINS, somado ao valor do ICMS (Imposto sobre Circulação de Mercadorias e Serviços).

Ao realizar esse cálculo, encontra-se o valor que deverá ser cobrado do consumidor por KWh, que deverá ser multiplicado pela quantidade de $\mathrm{KWh}$ consumidas em determinado período para encontrar o valor da energia consumida (ANEEL 2008a; ANEEL, 2008b; ANEEL, 2008c). Deve-se incluir, neste valor, a taxa de Contribuição de lluminação Pública (CIP), que é instituída por Leis municipais, variando, portanto, de município para município. O valor total a ser pago pode ser representado pela seguinte equação (ANEEL 2008a; ANEEL, 2008b; ANEEL, 2008c):

Valor total da conta a ser paga $=($ valor a ser cobrado por $K W h$. quantidade consumida $)+C I P$ 
A partir de 2015, foi atribuído o adicional de bandeira. Elas são classificadas em três tipos de bandeiras: a verde, a qual indica condições favoráveis para a geração de energia, ou seja, nenhum adicional é aplicado; a bandeira amarela indica que as condições para a geração de energia são menos favoráveis, o que implica o acréscimo de $R \$ 2,50$, para cada $100 \mathrm{KWh}$ consumidos; e a bandeira vermelha, caso as condições de energia sejam precárias, resulta em um acréscimo de $\mathrm{R} \$ 5,50$ por $100 \mathrm{KWh}$ consumidos, com base no ano da implementaçãovl.

\subsection{Breve revisão sobre a Regulamentação de energia no Rio Grande do Sul}

O contrato de concessão da AES Sul e da RGE foram assinados em 1997, que autoriza a exploração de energia elétrica por 30 anos. Já o contrato de concessão da CEEE foi assinado em 2000. As regulamentações das três distribuidoras são muito similares, nas quais ressaltam a importância de um serviço de qualidade e o comprometimento do aproveitamento hidrelétrico, principalmente no que condiz à CEEE.

A concessionária terá liberdade para direcionar seus negócios e investimentos. Ela deverá empregar tecnologias, mão de obra e instalações de qualidade para promover eficiência, qualidade e segurança nos serviços prestados. Ela fica encarregada de reparar suas instalações ou ampliá-las para atender à demanda futura atentando-se a recomendações dos órgãos regulatórios. Ela, também, fica encarregada de fornecer energia para toda região, sem exclusão de áreas com baixas densidades ou com população de baixa renda, incluindo áreas rurais (MME, 1997a; MME, 1997b; MME, 2000).

A concessionária deve cumprir com as leis de proteção ao ambiente, bem como implementar medidas que auxiliem na conservação da energia e na utilização correta, sem incidir em excedentes (MME, 1997a; MME, 1997b; MME, 2000). Deverá atender a todos os requisitos fiscais, trabalhistas e previdenciário, e permitir que encarregados da fiscalização tenham completo acesso a arquivos, a documentos, a registros contábeis e econômicos, à legislação e a normas que possam transitar em obras, analisar equipamentos e instalações utilizadas e a qualidade da energia distribuída (MME, 1997a; MME, 1997b; MME, 2000). A fiscalização irá elaborar relatórios expondo suas observações sobre a concessionária. Deverá, também, prestar contas, anualmente, ao órgão regulador, como também aos cidadãos que utilizam os serviços prestados pela empresa (MME, 1997a; MME, 1997b; MME, 2000).

A contabilidade da concessionária deverá atender às normas da Classificação de Contas e ao Plano de Contas do Serviço Público de Energia Elétrica, tendo como obrigação registrar e apurar investimentos, custos de transmissão, de geração, de distribuição e de comercialização relativos a novas e a antigas instalações (MME, 1997a; MME, 1997b; MME, 2000). advertida.

Se a administração da concessionária descumprir algumas destas obrigações, poderá ser multada ou

\subsection{Literatura Empírica para o Brasil}

Muitos artigos estudam a elasticidade preço e renda da demanda por energia elétrica. Alguns se dedicam a analisar países, como o Brasil, outras regiões e estados, assim como aplicam diversas técnicas para estimar os parâmetros. Alguns estudos utilizam o método Ordinary Least Squares (OLS) para analisar a elasticidade preço e renda da demanda por energia elétrica, como é o caso dos estudos realizados por Modiano (1984) e Villareal e Moreira (2016).

O estudo realizado por Modiano (1984) analisou a elasticidade preço e renda da demanda por energia elétrica residencial, comercial e industrial para o Brasil. Como o foco deste estudo é analisar a elasticidade preço da demanda residencial, iremos focar nos resultados obtidos por residência. Ele conclui que a elasticidade preço da demanda por energia elétrica residencial de curto prazo é dada por -0.118 , enquanto a de longo prazo corresponde a -0.403 , e a elasticidade renda de curto prazo é dada por 0.332 e a de longo prazo é 1.13.

Já Villareal e Moreira (2016) analisaram a elasticidade preço da demanda da energia elétrica para domicílios brasileiros. O período de estudo se baseia em dados de 1985 a 2013, e foram utilizadas regressões lineares para a identificação dos parâmetros. As elasticidades preço e as elasticidades renda de longo prazo encontradas foram de -0.23 e 01.88 , respectivamente.

Outros estudos utilizam os Modelos de Correção de Erros, ou seja, os modelos de Vetores Alto Regressivos (VAR) e Vetor de Correção de Erros (VEC). Estudos como o de Viana e Silva (2014), Schmitd e Lima (2004) e Andrade e Lobão (1997) utilizam esses modelos para estimar os parâmetros das elasticidades para o Brasil.

De acordo com o estudo de Viana e Silva (2014), a elasticidade preço de longo prazo corresponde a 0.707 e a elasticidade renda de longo prazo corresponde a 1.79. Já Schmitd e Lima (2004) identificaram a elasticidade preço e renda de longo prazo para o Brasil, para as três classes consumidoras. Eles encontraram que a elasticidade preço de longo prazo é -0.085, e a elasticidade renda de longo prazo corresponde a 0.539 . 
O estudo de Andrade e Lobão (1997) analisa o consumo de energia elétrica no Brasil e busca estimar as elasticidades preço e renda da demanda para os anos de 1963 a 1995. A elasticidade preço da demanda por energia elétrica residencial de curto e longo prazo corresponde a 0.06 e 0.212 , respectivamente. Enquanto a elasticidade renda da demanda por energia elétrica residencial de curto e longo prazo corresponde a 0.051 e 0.2132 , respectivamente.

Outros estudos buscam estimar a elasticidade preço e demanda por energia elétrica residencial para microrregiões, como estados. Uhr, Uhr e Chagas (2017b) estimaram a elasticidade preço e renda para a região metropolitana de São Paulo, utilizando dados desagregados entre o período de 1998 e 2008. Para estimar os parâmetros, foram utilizados dados em painel com controle de efeitos fixos. Os resultados encontrados mostram que a elasticidade preço da demanda por energia elétrica residencial varia entre $-0,26$ a -0,64. Entretanto, a elasticidade renda da demanda por energia elétrica residencial varia 0,11 e 0,32 . Outro estudo realizado por Uhr, Uhr e Chagas (2017a) analisa a elasticidade preço e renda por energia elétrica residencial para estados brasileiros. Para identificação das elasticidades, foi utilizado o método dos momentos generalizados (GMM). Os resultados identificados compreendem a variação dos valores -0.13 a -0.18 para a elasticidade preço de curto prazo, -0.62 a -1.47 para o longo prazo, e 0.08 a 0.12 para a elasticidade renda de curto prazo e 0.32 a 1.09 para longo prazo.

Já Irffi et al (2009) estimaram a demanda por energia elétrica para a Região Nordeste do Brasil, entre os anos de 1970 a 2003. Para a estimação dos parâmetros, foi utilizado o método de OLS Dinâmico, também conhecido como DOLS, e a mudança de regime. Os resultados obtidos, neste estudo, constatam que a elasticidade preço da demanda residencial de curto prazo é -0.2078 e a de longo prazo consiste em -0.687, e a elasticidade renda da demanda por energia elétrica residencial é no curto prazo 0.013 e no longo prazo 0.684 .

Também, foram utilizados os métodos de correções de erros VAR e VEC, para a estimação da energia elétrica em estados. Para analisar a elasticidade preço e renda, Siqueira et al (2006) utilizaram o modelo VAR e VEC para estimar os parâmetros da elasticidade. A elasticidade de curto prazo para o preço foi de $-0.298 \mathrm{e}$ de longo prazo corresponde a -0.412 , já a elasticidade renda estimada foi 0.181 para o curto prazo e 1.40 para o longo prazo. Mattos e Lima (2005) também utilizaram os métodos VAR e VEC para a estimação das elasticidades de longo prazo. $O$ objetivo do estudo foi estimar a elasticidade preço e renda por energia elétrica residencial para o estado de Minas Gerais nos anos de 1970 a 2002. Os resultados obtidos consistem na elasticidade preço de -0.258 e na elasticidade renda de 0.532 .

Em resumo, a literatura nacional identifica a elasticidade preço da demanda por energia elétrica residencial conforme a Tabela 1.

Tabela 1 - Estudos empíricos para o Brasil e cálculo das elasticidades

\begin{tabular}{|c|c|c|c|c|c|c|c|}
\hline \multirow[t]{2}{*}{ Autor } & \multirow[t]{2}{*}{ Período } & \multirow[t]{2}{*}{ Nível } & \multirow[t]{2}{*}{ Método } & \multicolumn{2}{|c|}{ Curto Prazo } & \multicolumn{2}{|c|}{ Longo Prazo } \\
\hline & & & & Preço (-) & Renda & Preço (-) & Renda \\
\hline $\begin{array}{l}\text { UHR; UHR; CHAGAS, } \\
\text { 2017b }\end{array}$ & $1998-2013$ & Estado & $\mathrm{FE}$ & $0.26-0.64$ & $0.11-0.32$ & - & - \\
\hline $\begin{array}{l}\text { UHR; UHR; CHAGAS, } \\
2017 a\end{array}$ & 2004-2014 & Estado & GMM & $0.13-0.18$ & $0.08-0.12$ & $0.62-1.47$ & $0.32-1.09$ \\
\hline $\begin{array}{l}\text { VILLAREAL; MOREIRA, } \\
2016\end{array}$ & $1985-2013$ & País & OLS & - & - & 0.230 & 0.188 \\
\hline VIANA; SILVA, 2014 & $1975-2006$ & País & VAR/VEC & - & - & 0.707 & 1.79 \\
\hline IRFFI et al., 2009 & $1970-2003$ & $\mathrm{RN}$ & DOLS & 0.2078 & 0.013 & 0.687 & 0.684 \\
\hline SIQUEIRA et al., 2006 & $1970-2003$ & RN & VEC & 0.298 & 0.181 & 0.412 & 1.40 \\
\hline MATTOS; LIMA, 2005 & 1979-2002 & MG & VAR/VEC & . & - & 0.258 & 0.532 \\
\hline SCHMITD; LIMA, 2004 & 1969-1999 & País & VAR/VEC & - & - & 0.085 & 0.539 \\
\hline ANDRADE; LOBÃO, 1997 & 1963-1995 & País & VAR/VEC & 0.06 & 0.212 & 0.051 & 0.2132 \\
\hline MODIANO, 1984 & $1963-1981$ & País & OLS & 0.118 & 0.332 & 0.403 & 1.13 \\
\hline
\end{tabular}

Fonte: elaborado pelos autores

Todos os resultados apresentados são consistentes entre si, ou seja, estão de acordo com outros estudos empíricos que estudam a elasticidade preço e renda da demanda por energia elétrica residencial.

\subsection{Literatura Empírica Internacional}

Além dos estudos realizados para o Brasil, há diversos estudos internacionais que buscam analisar a elasticidade preço e renda da energia elétrica. Eles utilizam diversos modelos econométricos para estimar as elasticidades. 
O estudo realizado por Filippini e Pachauri (2004) e por Zhou e Teng (2013) utilizaram o método OLS para estimar as elasticidades preço e renda da demanda por energia elétrica. O objetivo da pesquisa de Filippini e Pachauri (2004) era analisar a elasticidade preço e renda da demanda por energia elétrica em cerca de 30.000 famílias da Índia entre os anos de 1993 a 1994. Os resultados encontrados consistem que a elasticidade preço e renda da demanda de curto prazo são -0.29 a -0.51 e 0.60 a 0.64 , respectivamente.

Zhou e Teng (2013) analisou dados de pesquisas domiciliares urbanas da província de Sichuan; na China, os dados compreendem o período de 2007 a 2009. Ele concluiu que a elasticidade preço da demanda de curto prazo compreende uma variação correspondente a -0.35 a -0.50 , e a elasticidade renda de curto prazo corresponde à variação de 0.14 a 0.33 , sendo ambas inelásticas.

Alguns estudos utilizam o método dos momentos generalizados (GMM). Estudos como os realizados por Alberini e Filippini (2011), Okajima e Okajima (2013) e Liu (2004) utilizam esses modelos para estimar os parâmetros das elasticidades. Os resultados encontrados por Alberini e Filippini (2011) para a elasticidade preço da demanda por energia elétrica de curto prazo corresponde à variação de -0.08 a -0.15 , enquanto a de longo prazo se dá pela variação -0.45 a -0.75 para 48 estados dos Estados Unidos entre os anos de 1995 a 2007.

O intuito da pesquisa realizada por Okajima e Okajima (2013) é estimar a elasticidade preço da demanda por energia elétrica residencial para o Japão entre os anos de 1990 e 2007. Os resultados obtidos mostram que a elasticidade preço da demanda de curto prazo foi de -0.40 , enquanto a de longo prazo foi de -0.49. Já Liu (2004) estimou a elasticidade renda e preço da demanda por energia elétrica para os setores residenciais e industriais para os países da OCDE para os anos de 1978 a 1999. Atentando-se aos resultados do setor residencial, a elasticidade e renda da demanda de energia elétrica de curto prazo foi de -0.03 e 0.058 , respectivamente, enquanto as elasticidades preço e renda de longo prazo foi de -0.157 e 0.303 , respectivamente.

Além do OLS e GMM, outros métodos também são empregados para estimar a elasticidade preço da demanda, como os métodos LSDV (least squares dummy variable), QES (quadratic expenditure system), TVP (time-varying parameter), ARDL (autoregressive-distributed lag), entre outros.

Filippini (2011) analisou a elasticidade preço da demanda por energia elétrica para 22 cidades suíças para o período de 2000 a 2006. Ele utilizou o método LSDV (least squares dummy variable) e encontrou que a elasticidade preço da demanda varia entre -0.65 e -0.84 no curto prazo e - -1.27 a -2.26 no longo prazo. Já Schulte e Heindl (2017) estimou a elasticidade preço e renda da demanda por energia elétrica para a Alemanha durante os anos de 1993 a 2008, utilizando o modelo QES (quadratic expenditure system); os resultados compreendem a variação de -0.43 a -0.50 para a elasticidade preço da demanda e 0.4 a 0.41 para a elasticidade renda da demanda.

A pesquisa desenvolvida por Wang e Mogi (2017) estima as elasticidades preço e renda da demanda residencial e industrial por energia elétrica no Japão, com dados anuais de 1989 a 2014. Utilizando o modelo TVP (time-varying parameter), os resultados consistem na variação da elasticidade preço da demanda de curto prazo de -0.46 a -0.68 e na variação da elasticidade renda da demanda no curto de prazo de 0.86 a 1.59 .

O estudo realizado por Nesbakken (1999) busca estimar a elasticidade preço da demanda de curto prazo para a Noruega, nos anos de 1990, 1993 e 1995 e encontrou que variação da elasticidade corresponde a -0.24 a -0.53 . Por fim, Halicioglu (2007) estimou o impacto da elasticidade preço e renda da demanda por energia elétrica na Turquia durante o período de 1968 a 2005, utilizando o modelo ARDL (autoregressivedistributed lag). Os resultados foram -0.33 para a elasticidade preço de curto prazo e -0.52 para a elasticidade preço de longo prazo.

Sintetizando, a elasticidade de preço e renda da demanda por energia elétrica de curto e longo prazo pode ser encontrada na Tabela 2.

Tabela 2 - Estudos Empíricos Internacionais e calcula das elasticidades

\begin{tabular}{|c|c|c|c|c|c|c|c|}
\hline \multirow[t]{2}{*}{ Autor } & \multirow[t]{2}{*}{ Período } & \multirow[t]{2}{*}{ País } & \multirow[t]{2}{*}{ Método } & \multicolumn{2}{|c|}{ Curto Prazo } & \multicolumn{2}{|c|}{ Longo Prazo } \\
\hline & & & & Preço (-) & Renda & Preço (-) & Renda \\
\hline ALBERINI; FILIPPINI (2011) & 1995-2007 & USA & GMM & $0.08-0.15$ & - & $0.45-0.75$ & - \\
\hline $\begin{array}{l}\text { FILIPPINI; PACHAURI } \\
\text { (2004) }\end{array}$ & 1993-1994 & Índia & OLS & $0.29-0.51$ & $0.60-0.64$ & 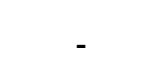 & - \\
\hline ZHOU; TENG (2013) & 2007-2009 & China & OLS & $0.35-0.50$ & $0.14-0.33$ & - & - \\
\hline OKAJIMA; OKAJIMA (2013) & 1990-2007 & Japão & GMM & 0.40 & & 0.49 & 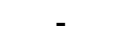 \\
\hline FILIPPINI (2011) & $2000-2006$ & Suíça & LSDV & $0.65-0.84$ & - & $1.27-2.26$ & 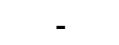 \\
\hline SCHULTE; HEINDL (2017) & 1993-2008 & Alemanha & QES & $0.43-0.50$ & $0.40-0.41$ & - & - \\
\hline LIU (2004) & 1978-1999 & OCDE & GMM & 0.03 & 0.058 & 0.157 & 0.303 \\
\hline HALICIOGLU (2007) & 1968-2005 & Turquia & ARDL & 0.33 & - & 0.52 & - \\
\hline NESBAKKEN (1999) & 1990/1993/1995 & Noruega & C.S. & $0.24-0.53$ & - & - & - \\
\hline WANG; MOGI (2017) & 1989-2014 & Japão & TVP & $0.46-0.68$ & $0.86-1.59$ & - & - \\
\hline
\end{tabular}

Fonte: Elaborado pelos autores. 


\section{BASE DE DADOS}

Este estudo utiliza dados em painel de 497 municípios do Rio Grande do Sul referentes aos anos de 2007 a 2015. Esses dados foram coletados da Agência Nacional de Energia Elétrica (ANEEL) e da Fundação de Economia e Estatística (FEE).

O Rio Grande do Sul possuía três principais distribuidoras de energia elétrica: a AES Sul Distribuidora de Energia, a Companhia Estadual de Energia Elétrica (CEEE) e a RGE Distribuidora Gaúcha de Energia, uma distribuidora da CPFL Energia. No ano de 2016, a AES Sul foi comprada pelo grupo CPFL Energia e passou a ser chamada de RGE Sul. Dado esSe acontecimento, o presente estudo analisou os dados que antecederam à compra.

AES Sul, fundada em 1997, atendia 118 municípios das regiões Metropolitanas e do Centro-oeste do estado, atendendo cerca de 1,3 milhão de unidades consumidoras (SME, 2016). A CEEE era responsável por atender a 72 municípios, incluindo a capital Porto Alegre e o litoral gaúcho (CEEE, 2015). Já a RGE foi fundada em 1998 e atendia a 254 municípios gaúchos da região norte e nordeste (ANEEL, 2008c), tornando-se a principal distribuidora de energia do estado.

Tabela 3 - Caracterização das Variáveis Utilizadas e das estatísticas descritivas

\begin{tabular}{clcccc}
\hline Variáveis & \multicolumn{3}{c}{ Caracterização } & Média & $\begin{array}{c}\text { Desvio- } \\
\text { Padrão }\end{array}$ \\
\hline KWH_Res & $\begin{array}{l}\text { Quantidade de KWh residencial consumido no } \\
\text { município. }\end{array}$ & $1.99 \mathrm{e}+09$ & $6.66 \mathrm{e}+08$ \\
Preço_Res & $\begin{array}{l}\text { Preço médio do KWh no município } \\
\text { PIBpc }\end{array}$ & $\begin{array}{l}\text { Produto Interno Bruto per capita deflacionado } \\
\text { municipal }\end{array}$ & $16.063,34$ & 11456,34 \\
\hline
\end{tabular}

Fonte: elaborado pelos autores.

As variáveis utilizadas para a determinação do consumo residencial foram a quantidade de $\mathrm{KWh}$ residencial consumido em cada município durante o período, o preço real médio do KWh residencial e o produto interno bruto per capita municipal. Tanto o preço quanto o produto interno foram deflacionados pelo IPCA (índice de preços ao consumidor amplo). Todas as variáveis são utilizadas em logaritmo natural.

\section{MÉTODO}

O objetivo desta pesquisa é identificar a elasticidade preço da demanda por energia elétrica residencial no Rio Grande do Sul; a relação linear da demanda por energia elétrica residencial pode ser descrita pela seguinte equação:

$$
\ln C_{m t}=\alpha+\beta_{1} \ln P_{m t}+\lambda_{m}+\lambda_{t}+\mu_{m t}
$$

$\mathrm{InC}_{\mathrm{mt}}$ é o logarítimo natural da quantidade de $\mathrm{KWh}$ consumidos no município $\mathrm{m}$, no período t. Já $\mathrm{InP} \mathrm{P}_{\mathrm{mt}}$ é o logaritmo natural do preço do KWh para o município $\mathrm{m}$, no período $\mathrm{t}$. $\mathrm{O}$ coeficiente a representa a constante, o coeficiente $\lambda_{\mathrm{m}}$ representa o efeito fixo municipal, e o coeficiente $\lambda_{t}$ representa os efeitos fixos temporais. Por fim, o termo de choque estocástico é representado por $\mu_{\mathrm{mt}}$.

Para atingir nosso objetivo, vamos explorar diversos estimadores que consideram a estrutura de dados em painel. Ou seja, aplicaremos os estimadores de dados em painel considerando dados empilhados (conhecidos como Pooled Ordinary Least Squares - POLS), o estimador de efeitos aleatórios (Random Effects - RE), o modelo de primeira diferença (First Difference - FD) e o modelo de efeitos fixos (Fixed Effects - FE). Esses estimadores possuem hipóteses distintas quanto ao efeito fixo individual. O modelo POLS é o modelo mais ingênuo, porque desconsidera por hipótese a existência das características fixas dos municípios para estimação. $\mathrm{O}$ modelo de RE pressupõe que existem características aleatórias dos municípios, entretanto essas características apenas afetam a variância dos termos de erro, desse modo, o estimador constrói uma matriz de variância e covariância, pondera as variáveis e procura identificar o estimador (esse processo é conhecido como Feaseble GLS). Cabe destacar que a consideração dos efeitos fixos individuais é importante porque estes podem ser fonte de endogeneidade. Se alguma das variáveis explicativas de um modelo econométrico é endógena, então, os coeficientes estimados serão enviesados e inconsistentes.

A literatura apresenta o teste de Hausman, o qual verifica se os efeitos fixos individuais afetam, de forma sistemática, a variável dos preços. Em caso positivo, os modelos os modelos FD e FE controlam esta fonte de endogeneidade. O efeito fixo não é a única fonte de endogeneidade para a variável de preços. Sendo assim, se existirem outras fontes de endogeneidade, uma solução é a utilização de variáveis instrumentais, isto é, variáveis que sejam correlacionadas com a variável endógena e que não sejam correlacionadas com 
o termo de erro. Isso possibilita uma variação exógena nos preços e implica identificação correta das elasticidades. Desse modo, podemos utilizar alguns instrumentos internos, tais como os preços defasados, ou a variação anual dos preços, e, até mesmo, o produto dessas como instrumentos.

Por fim, ocorreu um fato importante entre o ano de 2008 e 2009. A CEEE apresentou uma mudança na política de reajuste de preços, de modo que os preços aumentaram em, aproximadamente, $1 \%$, enquanto a AESSUL, que seguia uma tendência de ajustes igual à da CEEE para os anos anteriores, apresentou um aumento de, aproximadamente, $5 \%$ para o ano de 2009. Logo, podemos utilizar esse choque da mudança de preços da CEEE em comparação com a AESSUL como instrumento para identificação da elasticidade preço da demanda por energia elétrica residencial. Ou seja, podemos explorar as tendências paralelas dos preços da energia elétrica entre as empresas distribuidoras, e utilizar a mudança inesperada da política tarifária para determinada região como instrumento para os preços da energia elétrica. Assim, a equação dos preços é afetada da seguinte forma:

$$
\ln P_{m t}=\beta_{1} \text { Alteração }_{m}+\beta_{2} \text { Período }_{t}+\beta_{3} \text { Alteração }_{m} \cdot \text { Período }_{t}+\mu_{m t}
$$

Assim, consideramos a equação (08) como o primeiro estágio para a identificação da elasticidade preço da demanda na medida em que gera uma variação exógena sobre os preços, possibilitando a identificação do coeficiente, sem influências sistemáticas de uma eventual variável não observável.

\section{RESULTADOS}

Nesta seção, apresentamos os resultados da pesquisa em 3 Tabelas. A Tabela 4 apresenta os resultados das regressões para os diferentes estimadores de dados em painel. Os resultados em termos gerais segue aquilo que se espera segundo a teoria economica, isto é, o preço afeta, de forma negativa e significativa, a demanda por energia elétrica. Entretanto, precisamos aplicar alguns testes para endender qual o modelo mais adequado e quais os resultados mais confiaveis. Nesse sentido, devemos examinar, primeiramente, o teste de Breush-Pagan (1978) e o teste de Hausmann (1978). O teste de Breush-Pagan (1978) examina se a variância dos componentes individuais são zero. Analisando os valores encontrados, temos que, tanto na regressão (3) quanto na (4) Tabela 4, rejeita-se a hipótese nula de variância constante. Logo, concluímos que o estimador RE é preferível ao estimador POLS.

Tabela 4 - Elasticidade preço da demanda por energia elétrica residencial

\begin{tabular}{|c|c|c|c|c|c|c|c|c|}
\hline & $\begin{array}{c}\text { POLS } \\
\text { (1) }\end{array}$ & $\begin{array}{c}\text { POLS } \\
\text { (2) }\end{array}$ & $\begin{array}{l}\text { RE } \\
\text { (3) }\end{array}$ & $\begin{array}{l}\text { RE } \\
\text { (4) }\end{array}$ & $\begin{array}{l}\text { FD } \\
\text { (5) }\end{array}$ & $\begin{array}{l}\text { FD } \\
\text { (6) }\end{array}$ & $\begin{array}{l}\text { FE } \\
\text { (7) }\end{array}$ & $\begin{array}{l}\mathrm{FE} \\
(8)\end{array}$ \\
\hline \multirow[t]{2}{*}{ Elasticidade } & $-1.38^{\star \star \star *}$ & $-3.67^{\star \star \star}$ & $-0.798^{* * t}$ & $-0.138^{* * * t}$ & $-0.233^{* \star *}$ & $-0.184^{\star \star \star}$ & $-0.798^{*+*}$ & $-0.105^{* * *}$ \\
\hline & $(0.11)$ & $(0.23)$ & $(0.005)$ & $(0.018)$ & $(0.004)$ & $(0.013)$ & $(0.006)$ & $(0.017)$ \\
\hline EF. Municipal & Não & Não & Não & Não & Sim & $\operatorname{Sim}$ & Sim & Sim \\
\hline EF. Temporal & Não & Sim & Sim & Sim & Não & Sim & Não & Sim \\
\hline Hausman & & & & & & & 14.63 & 1523.62 \\
\hline Breush-Pagan & & & 6356.82 & 6272.35 & & & & \\
\hline$N$ & 3.883 & 3.883 & 3.883 & 3.883 & 3.387 & 3.387 & 3.883 & 3.883 \\
\hline
\end{tabular}

Fonte: Elaborado pelos autores. Notas: Os valores em parênteses são os desvios-padrão dos coeficientes. Os símbolos ${ }^{*},{ }^{* *} \mathrm{e}^{* * *}$ representam significância estatística de $10 \%, 5 \%$ e $1 \%$, respectivamente.

Já o teste de Hausman (1978) apresenta os valores 14.63 e 1523.62 para os modelos (7) e (8), então, rejeitamos a hipótese de que os efeitos individuais são não correlacionados com qualquer regressor no modelo. Logo, o mais indicado é o modelo que considera o controle dos efeitos fixos individuais. Como o painel é curto, isto é, a dimensão temporal é menor que a dimensão dos indivíduos, consideramos o estimador FE em detrimento do estimador FD.

Assim, os resultados dos modelos FE das colunas (7) e (8) são os candidatos mais adequados. E os resultados -0.798 e -0.105 são os mais confiáveis nesse primeiro momento. Entretanto, esses resultados carecem de tratamento e permanecem ingênuos porque não controlam outras possíveis fontes de endogeneidade.

A Tabela 5 apresenta as elasticidades para os estimadores de efeito fixo, considerando instrumentos internos. Conforme comentado anteriormente, utilizamos como instrumentos os preços defasados, ou a variação anual dos preços da energia elétrica, e, por fim, utilizamos, também, o produto destes como instrumentos. Em termos gerais, a Tabela 5 apresenta as regressões, considerando, apenas, o modelo de dados em painel com controles para efeitos fixos (modelo FE) e, adicionalmente, controlamos a possível endogeneidade reversa através de instrumentos internos. Ou seja, consideramos tanto a variável de preços 
defasada quanto a variação anual como instrumentos. As estimações (1) e (2) apresentam os resultados para as regressões como defasagem dos preços como instrumento. As regressões (3) e (4) apresentam as regressões com a variação dos preços como instrumento. As regressões (5) e (6) apresentam as regressões com ambas as variáveis instrumentais. Já as regressões (7) e (8) consideram ambos os instrumentos, e inclusive o seu produto.

Tabela 5 - Elasticidade preço da demanda por energia elétrica residencial

\begin{tabular}{|c|c|c|c|c|c|c|c|c|}
\hline & $\begin{array}{l}\text { FE-IV } \\
\text { (1) }\end{array}$ & $\begin{array}{c}\text { FE-IV } \\
\text { (2) }\end{array}$ & $\begin{array}{c}\text { FE-IV } \\
\text { (3) }\end{array}$ & $\begin{array}{c}\text { FE-IV } \\
\text { (4) }\end{array}$ & $\begin{array}{l}\text { FE-IV } \\
\text { (5) }\end{array}$ & $\begin{array}{c}\text { FE-IV } \\
(6)\end{array}$ & $\begin{array}{c}\text { FE-IV } \\
(7)\end{array}$ & $\begin{array}{c}\text { FE-IV } \\
(8)\end{array}$ \\
\hline \multirow[t]{2}{*}{ Elasticidade } & $-0.972^{* * * t}$ & $0.20^{* * *}$ & $-0.904^{* *+*}$ & $-4.11^{* * *}$ & $-0.706^{* t+t}$ & $-0.16^{\text {**t }}$ & $-0.70^{* * *}$ & $-0.145^{\star * t}$ \\
\hline & $(0.015)$ & $(0.043)$ & $(0.092)$ & $(0.748)$ & $(0.004)$ & $(0.008)$ & $(0.003)$ & $(0.008)$ \\
\hline EF. Municipal & Sim & Sim & Sim & Sim & Sim & Sim & Sim & Sim \\
\hline EF. Temporal & Não & Sim & Não & Sim & Não & Sim & Não & Sim \\
\hline p. F-test & $<0.001$ & $<0.001$ & 0.0001 & $<0.001$ & $<0.001$ & $<0.001$ & $<0.001$ & $<0.001$ \\
\hline$N$ & 3.387 & 3.387 & 3.387 & 3.387 & 3.387 & 3.387 & 3.387 & 3.387 \\
\hline
\end{tabular}

Fonte: elaborado pelos autores. Notas: Os valores em parênteses são os desvios-padrão dos coeficientes. Os símbolos ${ }^{*},{ }^{* *} \mathrm{e}^{* * *}$ representam significância estatística de $10 \%, 5 \%$ e $1 \%$, respectivamente.

Em termos gerais, os resultados permanecem, estatisticamente, significativos e negativos em todos os modelos. $\mathrm{O}$ teste $\mathrm{F}$ de força para os instrumentos internos mostra que todas as estratégias são adequadas. Os modelos das colunas de 5 a 8 apresentam o maior número de instrumentos, e a magnitude dos coeficientes fica entre -0.70 e -0.145 . Entretanto, o uso de instrumentos internos não consegue trazer certeza quanto à identificação correta da elasticidade preço da demanda por energia elétrica.

Assim, o último modelo empírico considera uma estrutura em dois estágios. No primeiro estágio, utilizamos o choque da mudança de preços da CEEE em comparação com a AESSUL para gerar uma mudança inesperada nos preços entre os consumidores (equação 08). No segundo estágio, fazemos a regressão descrita pela equação (07) da seção de método. Os resultados mostram que o efeito do aumento de preços da energia elétrica residencial em $1 \%$ afeta, negativamente, a demanda em $0,46 \%$, ou seja, a demanda por energia elétrica residencial é inelástica ao preço.

Tabela 6 - Elasticidade preço da demanda por energia elétrica residencial

\begin{tabular}{lc}
\hline & FE-2SLS \\
\hline Elasticidade & $-\mathbf{0 . 4 6}$ \\
& $(0.141)$ \\
\hline EF. Municipal & Sim \\
EF. Temporal & Sim \\
p. F test & $<0.001$ \\
$N$ & 400 \\
\hline
\end{tabular}

Fonte: elaborado pelos autores. Notas: Os valores em parênteses são os desvios-padrão dos coeficientes. Os símbolos ${ }^{*},{ }^{* *} \mathrm{e}{ }^{* * *}$ representam significância estatística de $10 \%, 5 \%$ e $1 \%$, respectivamente.

Os resultados da Tabela 6 são os resultados mais fortes porque consideram um instrumento exógeno externo à equação. Além disso, os resultados se aproximam muito daqueles encontrados pela literatura recente para o Brasil, principalmente, os trabalhos de Uhr, Uhr e Chagas (2017a), Uhr, Uhr e Chagas (2017b), Mattos e Lima (2005) e Schmitd e Lima (2004).

\section{CONSIDERAÇÕES FINAIS}

O objetivo da pesquisa foi identificar a elasticidade preço da demanda por energia elétrica residencial para o Rio Grande do Sul. Então, utilizamos os dados municipais dos 497 municípios gaúchos entre os anos de 2007 a 2015. Usamos diversas estratégias de identificação baseadas na estrutura de dados em painel: (i) dados empilhados, (ii) efeitos aleatórios, (iii) efeitos fixos, (iv) efeitos fixos e variáveis instrumentais internas, e, por fim, (v) efeitos fixos com variável instrumental externa.

Os resultados, considerando as estratégias de dados empilhados, efeitos aleatórios e efeitos fixos apresentaram elasticidades entre -0,105 e -3,4. O segundo conjunto de estratégias manteve o uso dos efeitos fixos, e adicionou os instrumentos internos de diversas formas. Os resultados variaram entre $-0,145$ e 0,9 . Por fim, a estratégia que considera os efeitos fixos e utiliza como instrumento externo a variação exógina sobre diferentes regiões nos preços mostra que o aumento de preços da energia elétrica residencial em $1 \%$ afeta, 
negativamente, a demanda em $0,46 \%$. Este último resultado é o mais forte em termos econométricos, e a magnitude do efeito aproxima-se muito à literatura nacional e internacional.

Este trabalho é importante porque apresenta novos resultados para a literatura de economia da energia no Rio Grande do Sul, principalmente, por utilizar diversas estratégias de identificação e identificar a elasticidade da demanda por energia elétrica para essa região do Brasil.

\footnotetext{
I http://www.ceee.com.br/pportal/ceee/Component/Controller.aspx?CC=3237

" https://www.cpfl.com.br/releases/Paginas/cpfl-energia-assume-a-operacao-da-aes-sul.aspx

III https://www.rge-rs.com.br/institucional/quem-somos/a-rge/Paginas/default.aspx

${ }^{\mathrm{IV}} \mathrm{https}: / / w w w . a n e e l . g o v . b r / s a l a-d e-i m p r e n s a-e x i b i c a o-2 /-$

/asset_publisher/zXQREz8EVIZ6/content/autorizado-o-reajuste-de-tarifas-das-distribuidoras-gauchas-aessul-e-rge/656877?inheritRedirect=false

$\checkmark$ http://www2.aneel.gov.br/aplicacoes/noticias/Output_Noticias.cfm? Identidade=3284\&id_area=90 vI

http://www2.aneel.gov.br/aplicacoes/noticias_area/arquivo.cfm?tipo=PDF\&idNoticia=8415\&idAreaNoticia=1
}

\section{REFERÊNCIAS}

AES Brasil. Relátorio de Sistentabilidade 2015. $2015 . \quad$ Disponível em http://aesbrasilsustentabilidade.com.br/upload/file/relatorio/relatorio_89_filept_brasil_rso2015_pt.pdf. Acesso em 02 de março 2020.

AES Sul - AES Sul Distribuidora Gaúcha de Energia S.A. Demonstrações contábeis: 31 de dezembro de 2015 com relatório dos auditores independentes sobre as demonstrações contábeis. Porto Alegre, 2016. Disponível em https://economia.estadao.com.br/fatos-relevantes/pdf/19002552.pdf. Acesso em 02 de março 2020.

AES Sul - AES Sul Distribuidora Gaúcha de Energia S.A. Relatório de Sustentabilidade. 2006. Disponível em http://www.aesbrasil.com.br/indicadores/2006/port/download/AES_Sul_RA_2006.pdf. Acesso em 03 de março 2020.

ALBERINI, Anna; FILIPPINI, Massimo. Response of residential electricity demand to price: The effect of measurement error. Energy Economics, 33 (5), 889-895, 2011.

ANDRADE, Thompson A. LOBÃO, Waldir J. A. Elasticidade renda e preço da demanda residencial de energia elétrica no Brasil. Instituto de Pesquisa Econômica Aplicada - IPEA. Rio de Janeiro. Texto para Discussão № 489 . Junho de 1997.

ANEEL - Agência Nacional de Energia Elétrica. Por dentro da conta de luz: informação de utilidade pública (AES Sul). Agência Nacional de Energia Elétrica. 4. Ed. - 32 p.: il. Brasília, 2008a. Disponível em https://www.aneel.gov.br/documents/656835/16505063/2008_PorDentroContaLuzAESSul.pdf/09be82b8-8448-ee027d50-cb56d30fbd22. Acesso em 02 de mar. 2020

ANEEL - Agência Nacional de Energia Elétrica. Por dentro da conta de luz: informação de utilidade pública (CEEE). Agência Nacional de Energia Elétrica. 4. Ed. - 32 p.: il. Brasília, 2008b. Disponível em https://www.aneel.gov.br/documents/656835/16505063/2008_PorDentroContaLuzCEEE.pdf/489f312c-3c3c-0481-f538692f30ca94f7. Acesso em 02 de mar. 2020

ANEEL - Agência Nacional de Energia Elétrica. Por dentro da conta de luz: informação de utilidade pública (RGE). Agência Nacional de Energia Elétrica. 4. Ed. - 32 p.: il. Brasília, 2008c. Disponível em https://www.aneel.gov.br/documents/656835/16505063/2008_PorDentroContaLuzRGE.pdf/8ab23743-6487-de84-fc96739a71b9248e. Acesso em 02 de mar. 2020

ANEEL - Agência Nacional de Energia Elétrica. Segundo Termo Aditivo. Contrato de Concessão no 12/97. Brasília, 2005a. Disponível

http://www2.aneel.gov.br/aplicacoes/Contrato/Documentos_Aplicacao/Segundo_Aditivo_AES_SUL_012_1997.pdf.

Acesso em 02 de março 2020.

ANEEL - Agência Nacional de Energia Elétrica. Quarto Termo Aditivo. Contrato de Concessão no 13/97. Brasília, 2010 b. Disponível

em: https://www2.aneel.gov.br/aplicacoes/Contrato/Documentos_Aplicacao/Quarto_Aditivo_013_1997_RGE.pdf. Acesso em 02 de março 2020.

ANEEL - Agência Nacional de Energia Elétrica. Segundo Termo Aditivo. Contrato de Concessão no 25/2000. Brasília, 2010c. Disponível em: http://www2.aneel.gov.br/aplicacoes/Contrato/Documentos_Aplicacao/Segundo_Termo_Aditivo_081-1999.pdf. Acesso em 02 de março 2020.

ANEEL - Agência Nacional de Energia Elétrica. Terceiro Termo Aditivo. Contrato de Concessão no 12/97. Brasília, 2010a. Disponível em: http://www2.aneel.gov.br/aplicacoes/Contrato/Documentos_Aplicacao/Terceiro_Termo_Aditivo_012-1997.pdf. Acesso em 02 de março 2020. 
ANEL - Agência Nacional de Energia Elétrica. Segundo Termo Aditivo. Contrato de Concessão no 13/97. Brasília, 2005b. Disponível

http://www2.aneel.gov.br/aplicacoes/Contrato/Documentos_Aplicacao/Segundo_Aditivo_RGE_013_1997.pdf. Acesso em 02 de março 2020.

ANEL - Agência Nacional de Energia Elétrica. Primeiro Termo Aditivo. Contrato de Concessão no 25/2000. Brasília, 2005c. Disponível em: http://www2.aneel.gov.br/aplicacoes/Contrato/Documentos_Aplicacao/Primeiro_Aditivo_081_1999_CEEE.pdf. Acesso em 02 de março 2020.

CEEE - Centro Estadual de Energia Elétrica. Relatório Anual de Sustentabilidade 2015. Disponível em https://economia.estadao.com.br/fatos-relevantes/pdf/20017959.pdf. Acesso em 20 de fev. 2020.

FILIPPINI, Massimo. Short- and long-run time-of-use price elasticities in Swiss residential electricity demand. Energy Policy, 39 (10), 5811-5817, 2011.

FILIPPINI, Massimo; PACHAURI, Shonali. Elasticities of electricity demand in Urban Indian Households. Energy Policy, 32, 429-436, 2004

HALICIOGLU, Ferda. Residential electricity demand dynamics in Turkey. Energy Economics, 29, 199-210, 2007.

IRFFI, G. et al. Previsão da demanda por energia elétrica para classes de consumo na região Nordeste, usando OLS dinâmico e mudança de regime. Economia Aplicada. Vol.13, n.1, pp.69-98, 2009.

LIU, Gang. Estimating Energy Demand Elasticities for OECD Countries: A Dynamic Panel Data Approach. Discussion Papers No. 373, Statistics Norway, Research Department, pp.27, 2004.

MATTOS, Leonardo Bornacki de; LIMA, João Eustáquio de. Demanda residencial de energia elétrica em Minas Gerais: 1970-2002. Nova economia. Belo Horizonte, v. 15, n. 3, p. 31-52, dez. 2005 . Disponível em http://www.scielo.br/scielo.php?script=sci_arttext\&pid=S0103-63512005000300002\&lng=pt\&nrm=iso. Acesso em 17 fev. 2020.

MME - Ministério de Minas e Energia. Contrato de concessão no 12/97. Brasília, 1997a. Disponível em: http://www2.aneel.gov.br/aplicacoes/Contrato/Documentos_Aplicacao/12.pdf. Acesso em 20 fev. 2020.

MME - Ministério de Minas e Energia. Contrato de concessão no 13/97. Brasília, 1997b. Disponível em: http://www2.aneel.gov.br/aplicacoes/Contrato/Documentos_Aplicacao/CD9713RGE(Norte-Nordeste).pdf. Acesso em 20 fev. 2020.

MME - Ministério de Minas e Energia. Contrato de concessão no 25/2000. Brasília, 2000. Disponível em: http://www2.aneel.gov.br/aplicacoes/Contrato/Documentos_Aplicacao/081.pdf. Acesso em 20 fev. 2020.

MODIANO, Eduardo Marco. Elasticidade renda e preços da demanda de energia elétrica no Brasil. Departamento de Economia da PUC. Rio de Janeiro. Texto para Discussão, n. 68, 1984.

NESBAKKEN, Runa. Price sensitivity of residential energy consumption in Norway. Energy Economics, 21(6), 493$515,1999$.

OKAJIMA, Shigeharu; OKAJIMA, Hiroko. Estimation of Japanese price elasticities of residential electricity demand, 19902007. Energy Economics, 40, 433-440, 2013.

SCHMIDT, Cristiane Alkmin Junqueira; LIMA, Marcos A. M. A demanda por energia elétrica no Brasil. Revista Brasileira de Economia. Vol.58, n.1, pp.68-98, 2004.

SCHULTE, Isabella; HEINDL, Peter. Price and income elasticities of residential energy demand in Germany. Energy Policy, 102, 512-528, 2017.

SIQUEIRA, Marcelo Lettieri; CORDEIRO JÚNIOR, Herbetes de Hollanda; CASTELAR, Ivan. A demanda por energia elétrica no nordeste brasileiro após o racionamento de 2001-2002: previsões de longo prazo. Pesquisa e Planejamento Econômico 36, 1, 137-178, 2006.

SME, Secretaria de Minas e Energia do Estado do Rio Grande do Sul. Plano energético do Rio Grande do Sul 20162025. 2016. Disponível em: http://minasenergia.rs.gov.br/planoenergetico. Acesso em 20 fev. 2020.

UHR, Daniel de Abreu Pereira; UHR, Júlia Gallego Ziero. CHAGAS, André Luis Squarize. Demand for residential energy in Brazil revisited: a dynamic panel data approach. Working paper, 2017a.

UHR, Daniel de Abreu Pereira; UHR, Júlia Gallego Ziero; CHAGAS, André Luis Squarize. Estimation of price and income elasticities for the Brazilian household electricity demand. University of São Paulo (FEA-USP), 2017b.

VIANA, Gustav Ives Mendes Nicácio; SILVA, Alexandre Lima Marques. Um modelo para projeções para demanda por energia elétrica, 2009-2017 para o setor residencial no Brasil. Revista Brasileira De Energia, vol. 20, pg. 107-126, 2014.

VILLAREAL, Maria José Charfuelan; MOREIRA, João Manoel Losada. Household consumption of electricity in Brazil between 1985 and 2013. Energy Policy, vol. 96, pg 251-259, 2016.

WANG, Nan; MOGI, Gento. Industrial and residential electricity demand dynamics in Japan: How did price and income elasticities evolve from 1989 to 2014? Energy Policy, 106, 233-243, 2017.

ZHOU, Shaojie; TENG, Fei. Estimation of urban residential electricity demand in China using household survey data. Energy Policy, 61, 394-402, 2013. 